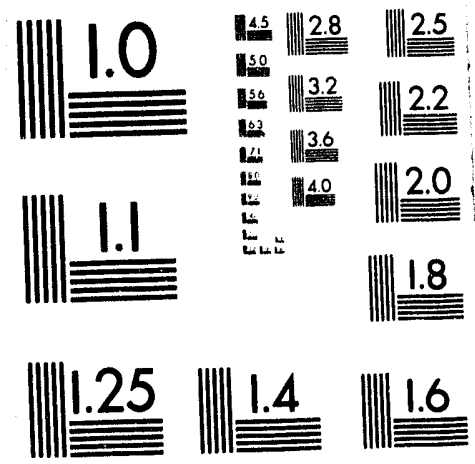



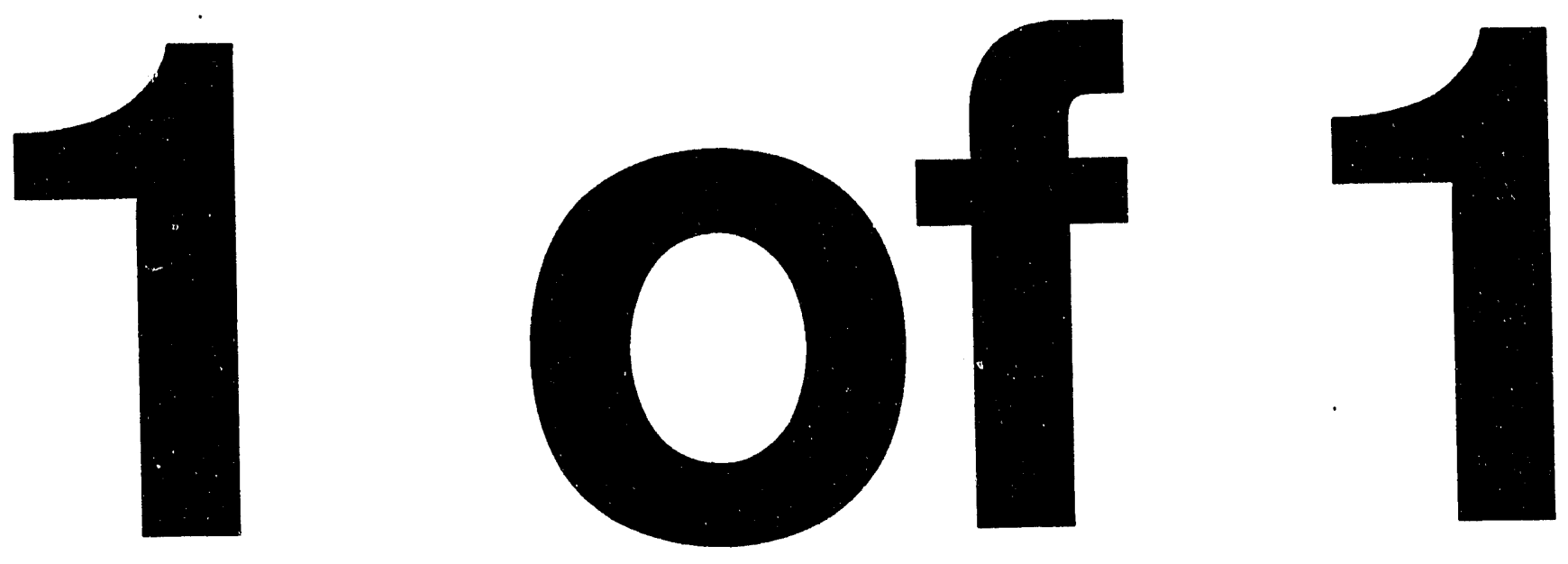


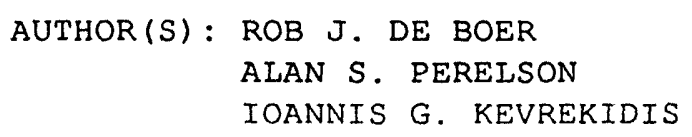

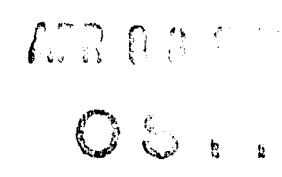

SUBMITTED TO: PROC. IFAC SYMPOSIUM ON MOLDELING AND CONTTROL IN BIOMEDICAL SYSTEMS MARCH 27-30, GALVESTON, TX

By acceptance of this axticle, the publisher recognizes that the U.S. Government retains a nonexclusive royalty-free license to publish or reproduce the published form of this contribution, or to allow other to do so, for the U.S. Government purposes.

The Los Alamos Natlonal Laboratory requests that the publisher ident lfy this article as wor: porformed uncer the auspices of the U.S. Department of Energy.

\section{LOS Alamos}

FORM NO. 836 R4

ST. NO. $26295 / 81$

MASTER

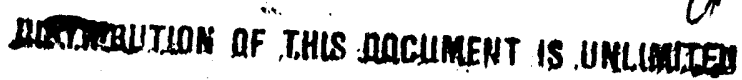




\title{
Immune Network Behavior: Oscillations, Chaos and Stationary States
}

\author{
Rob J. De Boer \\ Theoretical Biology, Utrecht University \\ Padualaan 8, 3584 CH Utrecht, The Netherlands \\ Alan S. Perelson \\ Theoretical Division, Los Alamos National Laboratory \\ Los Alamos, NM 87545, USA \\ and \\ Ioannis G. Kevrekidis \\ Department of Chemical Engineering and PACM \\ Princeton University, Princeton NJ 08544, USA
}

\begin{abstract}
We report two types of behavior in models of immune networks. The typical behavior of simple models, which involve B cells only, consists of several coexisting steady states. Finite amplitude perturbations may cause the model to switch between different equilibria. The typical behavior of more realistic models, which involve both B cells and antibody, consists of autonomous oscillations and/or chacs. While steady state behavior leads to easy interpretations in terms of immune memory, oscillatory behavior seems to be in better agreement with experimental data obtained in unimmunized animals.

The stability of the steady states, and the structure and interactions of the stable and unstable manifolds of the saddle-type equilibria turn out to be factors influencing the model's behavior. Whether or not the model is able to attain any form of sustained oscillatory behavior, i.e., limit cycles or chaos, seems to be determined by (global) bifurcations involving the stable and unstable manifolds of the steady states.
\end{abstract}

\section{DISCLAIMER}

This report was prepared as an account of work sponsored by an agency of the United States Government. Neither the United States Government nor any agency thereof, nor any of their employees, makes any warranty, express or implied, or assumes any legal liability or responsibility for the accuracy, completeness, or usefulness of any information, apparatus, product, or process disclosed, or represents that its use would not infringe privately owned rights. Reference herein to any specific commercial product, process, or service by trade name, trademark, manufacturer, or otherwise does not necessarily constitute or imply its endorsement, recommendation, or favoring by the United States Government or any agency thereof. The views and opinions of authors expressed herein do not necessarily state or reflect those of the United States Government or any agency thereof. 


\section{Introduction}

Jerne [8] suggested that the lymphocytes of the immune system may be organized in the form of a network, with communication between cells occurring by so-called idiotypic interactions. We have formulated a series of increasingly realistic models of the smallest $B$ cell network possible, that is, one that only involves interactions between an idiotype and its anti-idiotype.

The main motivation for undertaking such a study is that in previous work $[1,2,14]$ we analyzed simple models lacking the distinction between $B$ cells and antibody. The behavior of these simplified models is dominated by a number of stable steady states. The presence of multiple attractors allowed us to interpret the model's behavior in terms of "virgin", "immune" and "suppressed" states. By switching from a virgin state, in which the $B$ cells are inactive, to an immune state, the models can account for the phenomenon of immunological memory. Conversely, in more complex models that take into account both B cells and antibody $[3,4,5,12]$ fluctuations were found to be the predominant mode of dynamical behavior. The fluctuations were either truly periodic or chaotic. Although the models had multiple steady states, for the parameter values thought to characterize immune networks these steady states were not stable. Additionally, experimental data suggest that idiotype concentrations may indeed fluctuate periodically or chaotically on a time scale of two cycles per month $[9,13]$.

\section{The AB Model}

We have analyzed a series of models differing with respect to the incorporation of antibodies, B cell maturation, and compartmentalization. The essential ideas of the models can be understood from our "AB model" in which we only consider $B$ cells and antibodies. We consider B cells that may become activated by antiidiotypic antibody, upon which they proliferate and differentiate into antibody secreting cells. Free antibody, produced by differentiated activated B cells, may activate anti-idiotypic $B$ cells, and may react with anti-idiotypic antibodies to form complexes. These complexes are removed from the system.

We consider two $B$ cell clones, $B_{1}$ and $B_{2}$, that activate each other by the antibodies, $A_{1}$ and $A_{2}$, which the $\mathrm{B}$ cells produce. The activation of $\mathrm{B}$ cells by antibody is assumed to follow a log bell-shaped activation curve,

$$
f(A)=\frac{A}{\theta-1+A} \frac{\theta}{\theta+A},
$$

where $\theta$ determines the width of the function. Log bell-shaped activation functions are intrinsic to the process of receptor crosslinking $[11,10]$. We here 
consider two B cell populations

$$
\begin{aligned}
& d B_{1} / d t=\sigma+B_{1}\left(\rho f\left(A_{2}\right)-1\right), \\
& d B_{2} / d t=\sigma+B_{2}\left(\rho f\left(A_{1}\right)-1\right),
\end{aligned}
$$

where $\sigma$ represents the source of $B$ cells from the bone marrow and $\rho$ is the maximum rate of cell division. The antibody concentrations obey

$$
\begin{aligned}
& d A_{1} / d t=\delta\left(B_{1} f\left(A_{2}\right)-A_{1}\right)-\mu A_{1} A_{2}, \\
& d A_{2} / d t=\delta\left(B_{2} f\left(A_{1}\right)-A_{2}\right)-\mu A_{1} A_{2},
\end{aligned}
$$

where $\delta$ is the ratio of the $B$ cell and antibody lifetimes, and $\mu$ is the rate at which complexes are removed. The equations are non-dimensional: the populations have been scaled with respect to the maximum of the bell-shaped function, and time has been scaled to the lifetime of the $B$ cells $[6,7]$. The representative parameter values that we use are $\theta=10, \delta=0.1, \sigma=1.48 \times 10^{-3}, \rho=2$ and $\mu=2$.

The model is symmetric with respect to the hyperplane $A_{1}=A_{2}$ and $B_{1}=$ $B_{2}$. Introducing a new coordinate system it is possible to pinpoint the symmetry more closely $[6,7]$.

\section{Results}

This model may have six steady states, four of which lie on the plane of symmetry. There is a stable symmetric "virgin" state in which all populations are small. We describe four "activated" states: a stable asymmetric "immune state" in which $A_{1} \gg A_{2}$, a stable asymmetric "suppressed" state in which $A_{2} \gg A_{1}$, and two symmetric saddle states. We call the latter two "HH" and "MM" states,

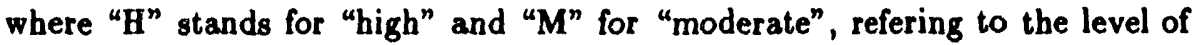
the corresponding population. Through numerical continuation, with $\theta$ as the bifurcation parameter, we show that four activated states are born from the virgin state by two saddle-node bifurcations and a pitchfork bifurcation. The immune and suppressed state become unstable at a subcritical Hopf bifurcation.

The typical behavior of the model is either stationary (for $\mu>12.6$ ), or periodic (for $\mu<12.6$ ), or apparently chaotic (for $0.2<\mu<14$ ). We show that the two saddle states are involved in a heteroclinic connection at $\mu \simeq 3$. The three-dimensional stable manifold of the lowest saddle point forms the separatrix between the virgin state and a state of "sustained activation" in the network. The latter corresponds to a form of immune memory. It either consists of stationary behavior in the immune or suppressed steady state, or of a stable limit cycle, or of a trajectory on an apparently chaotic attractor. 
We are interested in changes in this separatrix as a function of the parameters of the model. However, such a three-dimensional surface in four-dimensional space is too complex to visualize and analyze further. An alternative indication of changes in the structure and location of this three dimensional surface is provided by the one dimensional unstable manifold of the other saddle state. We found global bifurcations involving the one dimensional unstable manifold of the HH state and the stable manifold of the MM state. Since the unstable manifold of the HH state is just one dimensional, it lends itself for further numerical experiments and analysis and we use it as an indicator of the changes in the high dimensional basins of attraction.

\section{ACKNOWLEDGEMENTS}

This work was performed under the auspices of the U.S. Department of Energy, and was partially supported by NIH grant RR06555 and an NSF PYI award. The hospitality of the Center for Nonlinear Studies at Los Alamos National Laboratory and of the Santa Fe Institute are gratefully acknowledged.

\section{References}

[1] De Boer, R. J. 1988, Symmetric idiotypic networks: connectance and switching, stability, and suppression, in Theoretical Immunology, Part Two, (A. S. Perelson, Ed.), pp. 265-289, SFI Studies in the Science of Complexity, Vol. III, Addison-Wesley, Redwood City, CA.

[2] De Boer, R. J. and P. Hogeweg. 1989a, Memory but no suppression in lowdimensional symmetric idiotypic networks, Bull. Math. Biol. 51 pp.223-246.

[3] De Boer, R. J. and P. Hogeweg. 1989b, Unreasonable implications of reasonable idiotypic network assumptions, Bull. Math. Biol. 51 pp.381-408.

[4] De Boer, R. J., I. G. Kevrekidis, and A. S. Perelson. 1990, A simple idiotypic network model with complex dynamics, Chem. Eng. Sci. 45 pp.2375-2382.

[5] De Boer, R. J. and A. S. Perelson. 1991, Size and connectivity as emergent properties of a developing immune network J. Theor. Biol. 149 pp.381-424.

[6] De Boer, R. J., A. S. Perelson and I. G. Kevrekidis, 1993a, Immune network behavior I: From stationary states to limit cycle oscillations, Bull. Math. Biol. 55 pp.745-780.

[7] De Boer, R. J., A. S. Perelson and I. G. Kevrekidis, 1993b. Immune network behavior II: From oscillations to chaos and stationary states, Bull. Math. Biol. 55 pp.781-816. 
[8] Jerne, N. K. 1974, Towards a network theory of the immune system, Ann. Immunol. (Inst. Pasteur) $125 \mathrm{C}$ pp.373-389.

[9] Lundkvist, I., A. Coutinho, F. Varela and D. Holmberg. 1989, Evidence for a functional idiotypic network amongst natural antibodies in normal mice, Proc. Nat. Acad. Sci. USA. 86 pp.5074-5078.

[10] Perelson, A. S. 1981, Receptor clustering on a cell surface. III. Theory of receptor cross-linking by multivalent ligands: Description by ligand states, Math. Biosciences 53 pp.1-39.

[11] Perelson, A. S. and C. DeLisi. 1980, Receptor clustering on a cell surface. I. Theory of receptor cross-linking by ligands bearing two chemically identical functional groups Math. Biosci. 48 pp.71-110.

[12] Perelson, A. S. and G. Weisbuch. 1992, Modeling immune reactivity in secondary lymphoid organs Bull. Math. Biol. 54 pp.649-672.

[13] Varela, F. J., A. Anderson, G. Dietrich, A. Sundblad, D. Holmberg, M. Kazatchkine and A. Coutinho. 1991, The population dynamics of natural antibodies in normal and autoimmune individuals, Proc. Nat. Acad. Sci. 88 pp.5917-5921.

[14] Weisbuch, G., R. J. De Boer and A. S. Perelson. 1990, Localized memories in idiotypic networks J. Theor. Biol. 146 pp.483-499. 

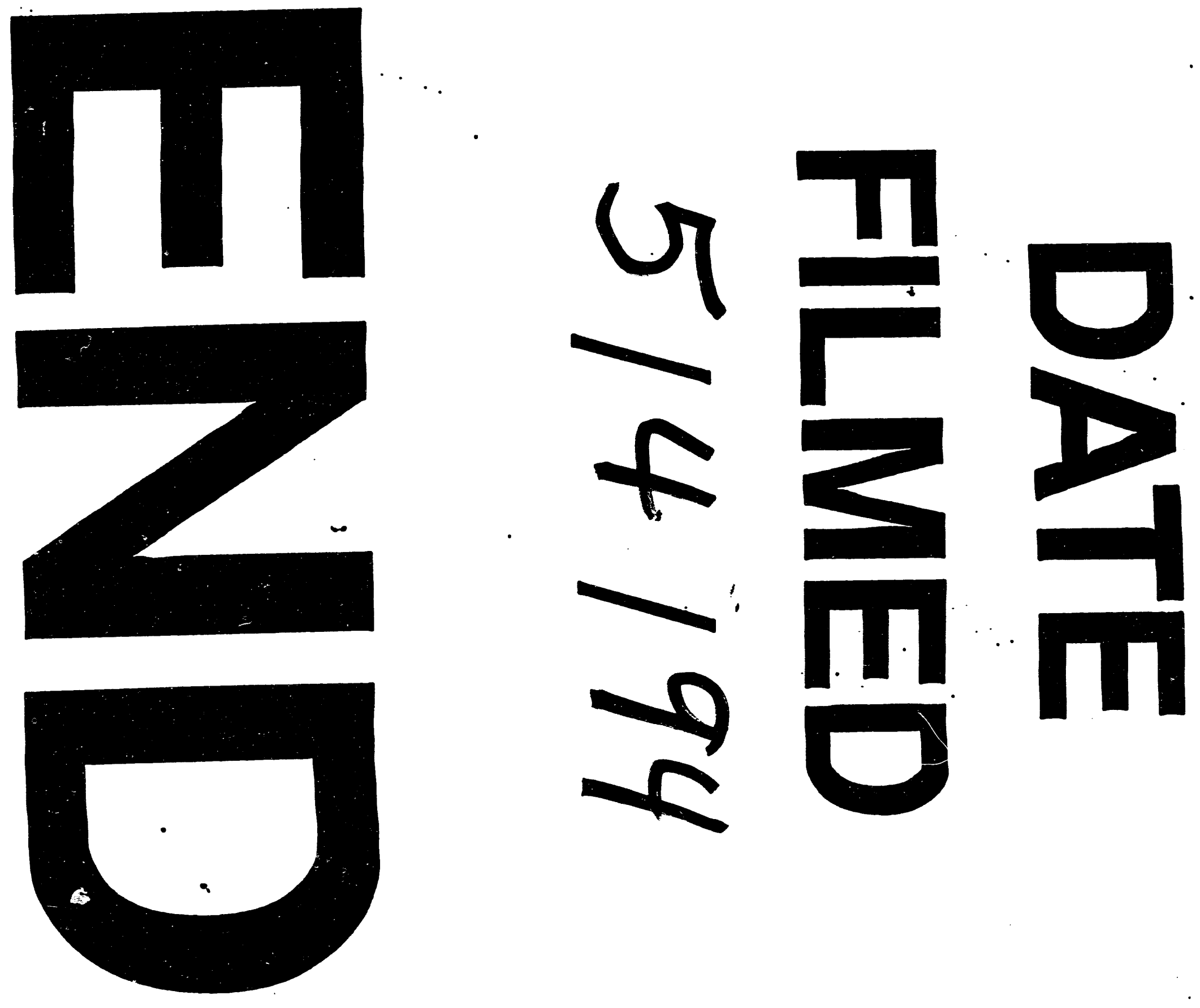
